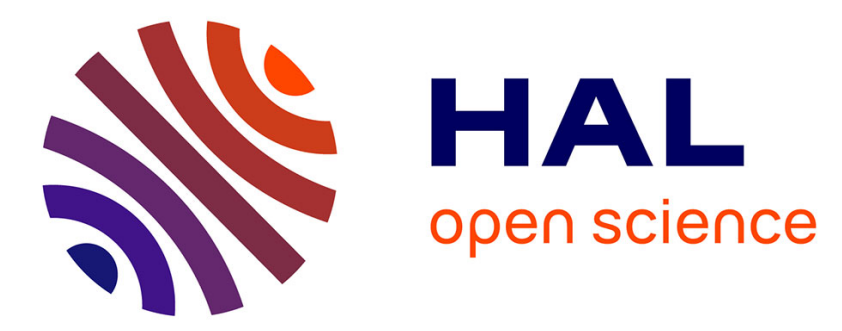

\title{
Magnification-continuous static calibration model of a scanning-electron microscope.
}

\author{
Abed Choaib Malti, Sounkalo Dembélé, Nadine Piat, Patrick Rougeot, \\ Roland Salut
}

\section{- To cite this version:}

Abed Choaib Malti, Sounkalo Dembélé, Nadine Piat, Patrick Rougeot, Roland Salut. Magnificationcontinuous static calibration model of a scanning-electron microscope.. Journal of Electronic Imaging, 2012, 21 (3), pp.033020-1 / 033020-12. 10.1117/1.JEI.21.3.033020 . hal-00772222

\section{HAL Id: hal-00772222 \\ https://hal.science/hal-00772222}

Submitted on 10 Jan 2013

HAL is a multi-disciplinary open access archive for the deposit and dissemination of scientific research documents, whether they are published or not. The documents may come from teaching and research institutions in France or abroad, or from public or private research centers.
L'archive ouverte pluridisciplinaire HAL, est destinée au dépôt et à la diffusion de documents scientifiques de niveau recherche, publiés ou non, émanant des établissements d'enseignement et de recherche français ou étrangers, des laboratoires publics ou privés. 


\section{Electronnic Imaging}

\section{SPIEDigitalLibrary.org/jei}

\section{Magnification-continuous static calibration model of a scanning-electron microscope}

Abed C. Malti

Sounkalo Dembélé

Nadine Le Fort-Piat

Patrick Rougeot

Roland Salut 


\title{
Magnification-continuous static calibration model of a scanning-electron microscope
}

\author{
Abed C. Malti \\ Université d'Auvergne \\ ALCoV-ISIT, UMR CNRS 6284 \\ 28 place Henri Dunant \\ 6300 Clermont-Ferrand, France \\ E-mail: abed.malti@gmail.com \\ Sounkalo Dembélé \\ Nadine Le Fort-Piat \\ Patrick Rougeot \\ Roland Salut \\ Université de Franche-Comté/ENSMM/UTBM \\ Femto-ST Institute, UMR CNRS 6174 \\ 24 rue Alain Savary \\ 25000 Besançon, France
}

\begin{abstract}
We present a new calibration model of both static distortion and projection for a scanning-electron microscope (SEM). The proposed calibration model depends continuously on the magnification factor. State-of-the-art methods have proposed models to solve the static distortion and projection model but for a discrete set of low and high magnifications: at low magnifications, existing models assume static distortion and perspective projection. At high magnifications, existing models assume an orthogonal projection without presence of static distortion. However, a magnification-continuous model which defines continuous switch from low to high magnifications has not yet been proposed. We propose a magnification-continuous static calibration model of the SEM. The static distortion and intrinsics of the projection matrix are modeled by partial differential equations (PDEs) with respect to magnification. The approach is applied with success to the JEOL-JSM 820 in a secondary electron imaging mode for magnification ranging from $100 \times$ to $10 \mathrm{k} \times$. The final RMS reprojection error is about 0.9 pixels. This result together with two application-based experiments: the consistent measurements of the bending of a cantilever and a 3-D reconstruction of a nano-ball emphasize the relevance of the proposed approach. (C) 2012 SPIE and IS\&T. [DOI: 10 .1117/1.JEl.21.3.033020]
\end{abstract}

\section{Introduction}

The scanning-electron microscope (SEM) imaging system is essential in the study of nanomaterials and micro-nanosystems. It allows us to observe, analyze, and manipulate these micro and nano specimens. However, the acquisition process is not free of phenomena which are necessary to model and to correct in order to proceed to vision-based applications: metrology, three-dimensional (3-D) reconstructions, visual servoing, etc. The first phenomenon is the time-dependent

Paper 12020 received Jan. 17, 2012; revised manuscript received Jul. 20, 2012; accepted for publication Jul. 27, 2012; published online Sep. 28, 2012.

0091-3286/2012/\$25.00 (C) 2012 SPIE and IS\&T drift which has received a particular interest. ${ }^{1}$ This drift is mainly due to the accumulation of electrons on the surface of the observed specimen. It can exceed hundreds of microns in an hour at $10 \mathrm{k} \times$ magnification with the JEOL-JSM 820. In this paper, we assume that the time-dependent pixel-drift is corrected with the method proposed in Ref. 1 The second phenomenon is the static distortion which can be defined as a systematic space distortion of the projected 3-D scene points. It is mainly due to the electromagnetic lens and the rastering process of the electron beam (the effect of these two distortion phenomena is depicted in Fig. 1). The third phenomenon is the mapping which relates a 3-D point in the observed specimen to its projection in the two-dimensional (2-D) image. This mapping depends on the magnification factor and can be either perspective or orthogonal (i.e., parallel). Sutton et al. ${ }^{2}$ have addressed this problem but for a discrete set of low and high magnifications. At low magnification, existing models assume only static distortion and perspective projection. At high magnification, existing models assume an orthogonal projection without presence of static distortion. These discrete calibration models are a real bottleneck during micro/nano-material inspection, characterization, or manipulation. Indeed, we often need to smoothly switch from one magnification factor to another to collect global and local information of the observed specimen. Unfortunately, with the existing models we are obliged to go through the set of precalibrated discrete set of magnifications.

This paper addresses the problem of modeling magnification-continuous parameters of the static distortion and the projection of the SEM. A systematic method of estimating the static distortion and the projective mapping in a continuous range of magnification scale is proposed. We assume that the dynamical pixel-drift is compensated as explained in Ref. 1. 


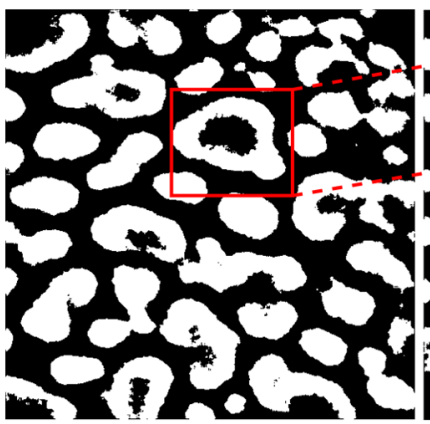

(a)

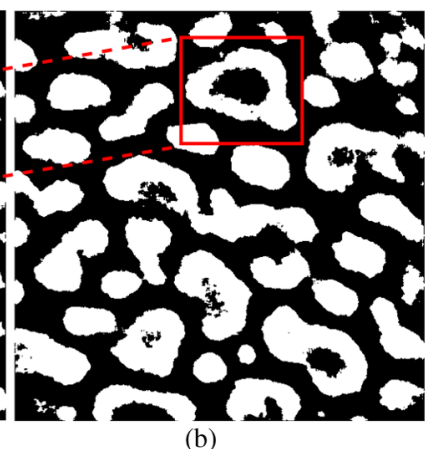

Fig. 1 The distortion phenomenon in image acquisition of a gold on carbon specimen by the JEOL-JSM 820 SEM. The image resolution is $512 \times 512$ pixels. (a) The initial acquired image. (b) After $15 \mathrm{~min}$, the patterns of the specimen have moved due to the time-dependent distortion effect.

\subsection{Related Work}

During the last decade, several authors have addressed the calibration problem of the SEM. They mainly investigated two aspects of the problem: 1. the dynamic calibration which includes the time-dependent pixel-drift and 2. the static calibration which includes static distortion and projection matrix calibration. The calibration of the pixel-drift has received the most particular attention in the electron microscopy community. These studies proved that the drift depends mainly on the time and the magnification when the magnification ranges from $100 \times$ to $10 \mathrm{k} \times{ }^{1}$ It is shown in Ref. 2 that for magnification higher than $10 \mathrm{k} \times$, the drift is space dependent and has to be estimated for each acquired pixel of the image. The drift was estimated first in full-image-based on digital image correlation (DIC) in Ref. 1 then in three other works in Refs. 2, 3, and 4. In Ref. 5 it was estimated in the frequency domain using fast Fourier transform (FFT). The drift trajectory flow over time has been modeled by means of B-spline curves fitting. ${ }^{1}$ Kalman filtering techniques also were tested and validated in the case of thermal drift calibration in scanning probe microscopy. ${ }^{6}$

The static calibration which includes static distortion calibration and projection calibration was modeled similarly as with classic optical imaging systems, ${ }^{7,8}$ Some authors observed a nonradial behavior of the static distortion and have addressed this problem using B-splines to fit the spatial field of evolution of distortion. Other authors assumeed a perspective projection in the case of low magnifications (up to $5 \mathrm{k} \times$ ) and an orthographic projection in the case of high magnifications (more than $5 \mathrm{k} \times$ ). ${ }^{1,9-11}$ This static calibration is usually processed for a discrete set of magnification ranging either in low or high magnification scales. For medium magnification scales, the literature did not provide a clear embedded model which allows us to switch smoothly from one model to another. However, in several applications we often need to go through different scales of magnification: (1) 3-D reconstruction of micro and nano scale specimens: ${ }^{9,12,13}$ in SEM imaging systems it is difficult to have the whole observed specimen in one single image. We need different images at different scales to have local information with details as well as global information of the observed specimen. In order to register this information to obtain a consistent 3-D shape with coarse and fine representation we need a magnification-continuous calibration model,

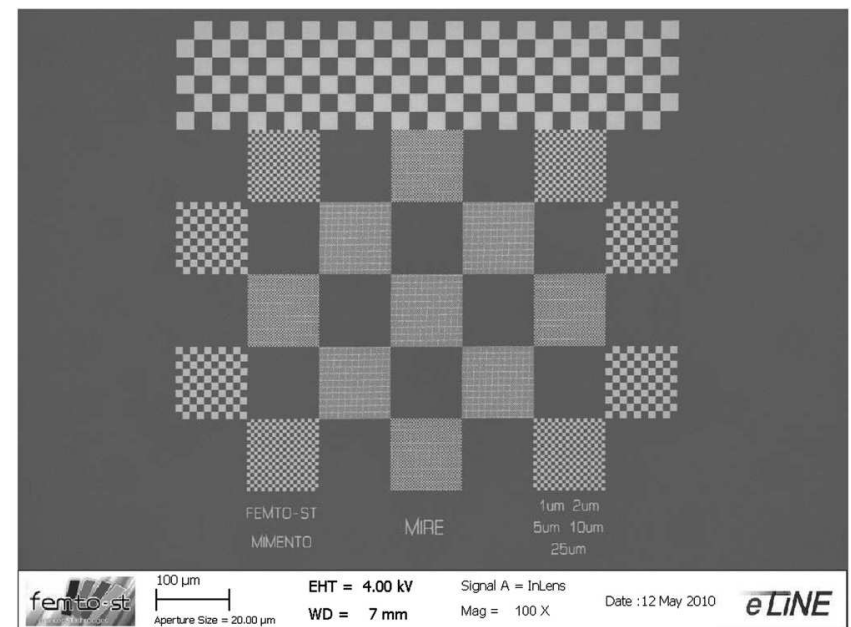

Fig. 2 Multi-scale calibration grid designed at FEMTO-ST lab. It contains multiple chess grid regions with squares going from $25 \mu \mathrm{m}$ down to $1 \mu \mathrm{m}$ per side.

(2) deformation measurement, ${ }^{14}$ (3) nanomaterials tracking, ${ }^{13,15,16}$ positioning and handling, ${ }^{17}$ and (4) micro-mobile robot positioning. ${ }^{18}$ In these different situations, a magnification-continuous calibration model will provide calibration parameters for every required magnification scale.

\subsection{Contribution}

A magnification-continuous static calibration model of the SEM is developed. It addresses the static calibration, i.e., the estimation of the spatial distortion and the geometric projection matrices. The calibration parameters are expressed into partial differential equations (PDEs) with respect to magnification. To cover a large magnification range, a specific calibration specimen is developed (see Fig. 2). It contains squares of various sizes enabling the calibration over a wide range of magnification. Images of this specimen are acquired for various magnifications and poses. In our study, we assume that the dynamical pixel-drift is compensated as explained in Ref. 1. The developed approach is applied to the JEOL-JSM 820 SEM for magnification ranging from $100 \times$ to $10 \mathrm{k} \times$. The RMS of the reprojection error is about 0.9 pixels. It shows up the accuracy of the proposed model. We propose two application-based experiments with real data to show the practical relevance of our model: 1. an accuracy deformation measurement experience at three different magnification scales 6450, 7760, and 8890 and 2. a 3-D reconstruction of nano-ball of $900 \mathrm{~nm}$ diameter. If a magnification-continuous calibration model is not required for single image of the observed specimen, it is of great importance when we need to register to the same reference frame multiple images of global and local views of an observed specimen.

\subsection{Structure and Notations}

This paper is organized as follows: Sec. 2 presents a brief technical description of the SEM. Section 3 describes the proposed approach for a multiscale static calibration. Section 4 presents the calibration results on the JEOL-JSM 820 and two real application experiments within discussion and comments. Finally, Sec. 5 concludes and draw future works. 
2-D points in homogeneous coordinates are denoted by symbols in typewriter font [e.g., $u=\left(u_{x}, u_{y}, 1\right)^{T}$ ]. 3-D points are indicated by plain letters [e.g., $\left.\mathbf{C}=\left(C_{x}, C_{y}, C_{z}\right)^{T}\right]$. Matrices are denoted by uppercase sans serif font (e.g., A). This notation is also adopted for $n$-dimensional vectors. However, vectors providing a direction in 3-D are represented using plain lowercase topped by an arrow (e.g., $\vec{l}$ ). For convenience, and given two $3 \times 1$ vectors $\vec{l}$ and $\vec{m}$, the dot product is indicated either using $\langle.,$.$\rangle or using regular$ matrix/vector multiplication (e.g., $\langle\vec{l}, \vec{m}\rangle=\vec{l}^{\top} \vec{m}$ ) and the cross product is carried either using the symbol $\times$ or using the skew-symmetric matrix (e.g., $\vec{l} \times \vec{m}=[\vec{l}]_{\times} \vec{m}$ ). For sake of simplicity, $\|\cdot\|_{2}$ denotes the vector norm 2 in any real vector space $\bullet^{n}$ of finite dimension $n$. The symbols $\mu \mathrm{m}$ and $\mathrm{nm}$ designate, respectively, micro-meter and nano-meter unit distances.

\section{Scanning-Electron Microscope}

\subsection{Technical Description}

Since the commercial availability of the SEM in 1966, it has been a valuable resource for viewing samples at a much higher resolution and depth of field than the typical light microscope. Similar to the light microscope, the SEM contains an illumination source. This source, known as the electron gun, supplies the electrons that form an electron beam, see Fig. 3. The shorter wavelength of electrons compared to visual light permits a resolution in the nano-meter range. As the electron beam proceeds down in the electron column toward the specimen chamber, lenses (usually magnetic) are used to focus the beam. After the beam has been focused by lenses, a pair of deflection coils scan the beam over a rectangular portion on the specimen substrate. ${ }^{19}$ The sample is typically mounted on a specimen stage which can be motion controlled with a joystick. The interior of the SEM contains a vacuum environment that supports beam formation and prevents the electrons from scattering. Detectors inside the chamber collect the emitted and/or scattered radiation from the specimen. Mostly secondary electrons (SE) are responsible for the topographic contrast in SEM images. This signal is then processed and used to display a 2-D image of the specimen on a computer.

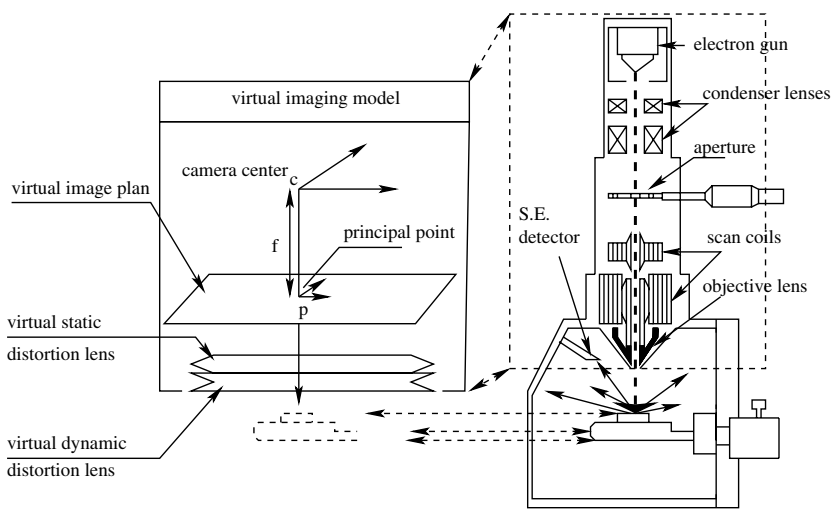

Fig. 3 The SEM and the corresponding modeling of the image acquisition process.

\subsection{Formal Imaging Model}

For magnifications ranging from $100 \times$ to $10 \mathrm{k} \times$, the imaging model of the SEM combines three functions:

1. a pixel-drift action which is both magnification and time-dependent,

2. followed by a static distortion action which is magnification-dependent,

3. followed by an action of projection. The law of projection to be applied depends on the magnification. While the scanning electron beam can be assumeed as central projective, it should be interpreted as parallel projective above a magnification of $1000 \times$ and more. ${ }^{1}$ The whole imaging model can be formalized as:

$$
\hat{\mathbf{x}}=\mathbf{f}_{t, g}^{d}\left[\mathfrak{f}_{g}^{s}\left(\mathrm{P}_{g} \mathbf{X}\right)\right]
$$

with $\mathbf{X}$ a $3-D$ scene point and $\hat{\mathbf{X}}$ the corresponding acquired image pixel. $\mathbf{f}_{t, g}^{d}$ and $\mathbf{f}_{g}^{s}$ are two dimensional functions (image $x$-axis and $y$-axis) which, respectively represents the dynamical drift and the static distortion. $\mathrm{P}_{g}$ is the matrix of projection of a 3-D point $\mathbf{X}$ of a given scene.

Henceforth, to retrieve a 3-D ray incident from a 3-D point of scanned scene the corresponding pixel is first corrected from the drift effect, then is statically undistorted and finally back-projected. In this work, the dynamical pixel-drift $\mathbf{f}_{t, g}^{d}$ is estimated and compensated as explained in Ref. 1.

\section{Magnification-Continuous Static Calibration Model}

The image-based static calibration includes static distortion calibration and image projection calibration. They are both time-independent but they are magnification-dependent. They are treated at the same time because usually the same calibration pattern is used for both and because of the dependance between the static distortion and the projection parameters. $^{20}$

\subsection{Static Distortion Calibration}

In SEM images, static distortions are mainly caused when the scan coils sweep the region to scan. In contrast with the dynamic distortion, at low magnification the static distortion is much more significant than at high magnification. ${ }^{1}$

\subsubsection{Distortion model}

The proposed spatial distortion model represents this physical phenomenon as a decentered shift which has both a radial and tangential component, ${ }^{21}$ (see Fig. 4):

$$
\mathbf{x}^{d}-\mathrm{e}=\underbrace{\frac{2}{1+\sqrt{1-4 \xi_{r} r_{u}^{2}}}\left(\mathbf{x}^{u}-\mathrm{e}\right)}_{\text {radial }}+\underbrace{\xi_{t}\left[r_{u}^{2}+2\left(x^{d}-x^{u}\right)^{2}\right]}_{\text {tangential }},
$$

where $\xi_{r}, \xi_{t}$ are the distortion parameters to be determined and $e$ is the center of distortion which is assumed to be different of the image center. $\lambda=2 / 1+\sqrt{1-4 \xi_{r} r_{u}^{2}}$ refers to the factor of distortion and depends on the square of the radius of distortion $r_{u}=\left\|\mathrm{X}^{u}-\mathrm{e}\right\|$ between the undistorted image point and the center of distortion. In the calibration 


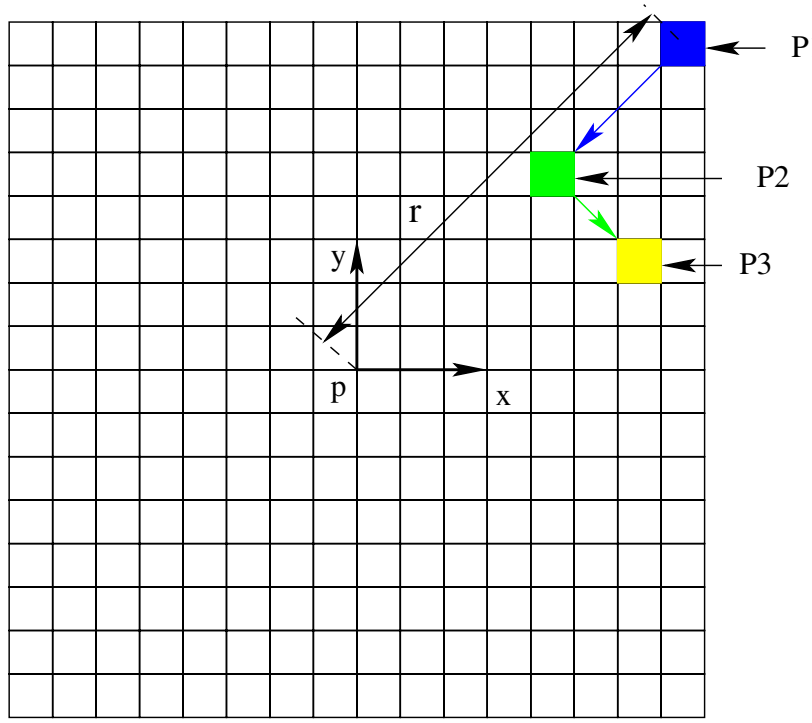

Fig. 4 Combination of radial and tangential distortion. In this example, we assume that the distortion is centered in the middle of the image. The original pixel $P 1$ moves radially toward the center to position $P 2$ than tangentially to $P 3$. Removing the static distortion defect is to recover $P 1$ starting from $P 3$. In our method, the center of distortion is estimated using a chess grid pattern.

of the distortion we first assume $\xi_{t}=0$ and estimate $e$. Then we initialize $\xi_{t}$ and $\xi_{r}$ to zero and estimate them using bundle adjustment methods. ${ }^{22}$ To see whether or not a given image undergoes distortion, finding the center of distortion is a good evaluation method. ${ }^{20}$ This procedure is described in the next paragraph.

\subsubsection{Determination of the center of distortion}

The importance of determining the center of distortion has long been recognized in the photogrammetry community. The method described in this paragraph was first proposed in. Ref. 20. The estimation of the center of distortion involves the use of a geometrically structured calibration pattern, see Fig. 5. It may consist on a plane with several distinguishable points $\left\{x_{i}^{c}\right\}_{i \in \bullet}$. The positions of the points $\mathbf{X}_{i}^{c}$ are assumed to be known in an Euclidean coordinate frame on the plane. Let $\mathbf{X}_{i}^{d}$ denote the corresponding points in the distorted image. The calibration pattern points $\mathbf{X}_{i}^{c}$ and the undistorted image points $x_{i}^{u}$ (in pixel coordinates) are related by a homography $\mathrm{H}$, according to $x_{i}^{u}=H x_{i}^{c}$. Note
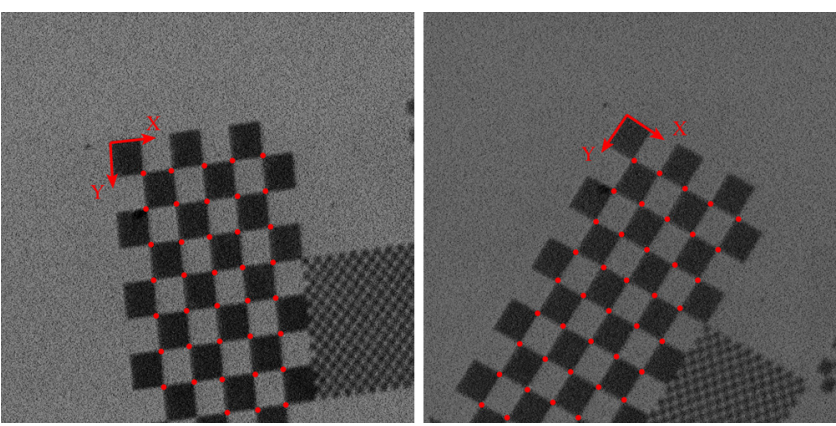

Fig. 5 An example of static distortion at $400 \times$ of magnification using the calibration pattern designed at FEMTO-ST lab. The image size is of $512 \times 512$ pixels and the size of the squares is of $25 \mu \mathrm{m}$ per side. The estimated center of distortion is at $\sim(290,300)^{T}$ pixels. that the superscripts $u, d$, and $c$, are used to distinguish the undistorted, distorted, and calibration points, where the subscript $i$ runs over all points. The points $x_{i}^{u}$ are next distorted radially away from the center of distortion e, to give

$$
\mathrm{x}_{i}^{d}=\mathrm{e}+\lambda_{i}\left(\mathrm{x}_{i}^{u}-\mathrm{e}\right)
$$

Note that the distortion factor $\lambda_{i}$ is typically different for each point. We multiply this expression on the left by $[e]_{X}$ (the skew-symmetric $3 \times 3$ matrix representing the cross product), resulting in:

$$
[\mathrm{e}]_{\mathbf{x}} \mathbf{x}_{i}^{d}=\lambda_{i}[\mathbf{e}]_{\mathbf{x}} \mathbf{x}_{i}^{u}
$$

where the terms e disappears when multiplied by $[e]_{\mathrm{x}}$. However, since $\mathrm{x}_{i}^{u}=\mathrm{Hx}_{i}^{c}$, we have:

$$
\left.{ }_{[\mathrm{e}}\right] \mathbf{x x}_{i}^{d}=\lambda_{i}[\mathrm{e}]_{\mathrm{x}} \mathrm{H} \mathbf{x}_{i}^{c} \text {. }
$$

Finally, multiplying on the right by $x_{i}^{d}$, and observing that $\mathbf{X}_{i}^{d}[\mathbf{e}]_{\mathbf{x}} \mathbf{X}_{i}^{d}=0$, because $[e]_{x}$ is skew-symmetric, we obtain:

$$
0=\lambda_{i} \mathbf{x}_{i}^{d}\left([e]_{x} \mathrm{H}\right) \mathbf{x}_{i}^{c} .
$$

Writing $F=[e]_{x} H$, we have the usual fundamental matrix relation:

$$
\mathbf{x}_{i}^{d} \mathrm{Fx}_{i}^{c}=0
$$

The matrix $\mathrm{F}$ may be called the fundamental matrix for radial distortion. It is a $3 \times 3$ matrix of rank 2 . It may be computed using, for instance, the 8-point algorithm ${ }^{7}$ from 8 grid points and their projections in the image plane. The center of radial distortion can be extracted as the left epipole. In the case where there is no radial distortion at all, the above computation of the fundamental matrix is unstable, and the estimated value of $e$ is essentially arbitrary and meaningless. The nature of this instability is related to the fact that in this case the point $x_{i}^{c}$ and its projection can be related only by a homography from the $3-\mathrm{D}$ space to the image plane. If there is no radial distortion, then it does not make much sense to talk about a center of distortion. Without radial distortion, the distortion factor $\lambda_{i}$ will equal unity for each point, and the distortion equation $\mathbf{x}_{i}^{d}=\mathrm{e}+\lambda_{i}\left(\mathbf{x}_{i}^{u}-\mathrm{e}\right)$ reduces to $\mathbf{x}_{i}^{d}=$ $\mathrm{x}_{i}^{u}$ independent of $\mathrm{e}$. This degenerate situation is easily detected during the computation of the fundamental matrix. This property allows us to determine whether there is or not a static distortion effect in SEM images when one goes through magnifications. ${ }^{20}$ Concretely, in the degenerate situation, the computation of the fundamental matrix with the 8-point algorithm will still produce a matrix $F$ satisfying $\mathbf{x}_{i}^{d} \mathbf{F} \mathbf{x}_{i}^{c}=0$. We decompose $\mathrm{F}$ in the form $\mathrm{F}=\mathrm{QH}$, where $\mathrm{H}$ is the homography relating the points $x_{i}^{c}$ and $x_{i}^{u}$, and $\mathrm{Q}$ is an arbitrary skew-symmetric matrix relating the points $x_{i}^{u}$ and $x_{i}^{d}$. In the degenerate case, points $\mathbf{X}_{i}^{d}$ and $\mathbf{x}_{i}^{c}$ also satisfy the relation $\mathrm{x}_{i}^{d}=\mathrm{x}_{i}^{u}=\mathrm{Hx}_{i}^{c}$ which is not the case in the nondegenerate situation. The detection of degenerate situations and computation of the center of distortion are outlined in Algorithm 1.

\subsection{Projection Model}

After compensating the image drift and undistorting the resulting pixels it remains to determine the projection 
Algorithm 1 Detection of degenerate situations and estimation of center of distortion.

Data: A set of $N_{c}$ grid points $x_{i}^{c}$ and their projections $x_{i}^{d}$ at a magnification $g$

1 Estimate the fundamental matrix $F$ using the 8-point algorithm; ${ }^{7}$

2 decompose matrix $F=Q H$ as product of homography $H$ and skew-symmetric matrix $Q$;

3 compute re-projection error $\varepsilon=\frac{1}{N_{c}} \sum_{i=1}^{N_{c}}\left\|x_{i}^{d}-H x_{i}^{c}\right\|_{2}$;

4 if $\varepsilon \leq \tau$ then

5 comments: we are in a degenerate situation (no distortion);

6 else

7 comments: we are in a nondegenerate situation;

8 compute the center of distortion $e_{g}$ as the left epipole of $F{ }^{7}$

9 end

Note: $\|\cdot\|_{2}$ denotes the 2-norm. We practically set the threshold to $\tau=10^{-3}$ pixels.

model. Because of the large range of magnification scales of the SEM, the projection model can vary from perspective to orthographic. Existing works ${ }^{11,23}$ assume either a perspective model for low magnification or an orthographic projection for high magnification with an abrupt switch at the magnification of transition which is experimentally determined (usually $5 \mathrm{k} \times$ ). Formally speaking, as the focal length increases and the distance between the image plane and the object decreases, the image remains the same size but perspective effect diminishes. The parallel projection can then be conceived as a double projection. All the object points are projected orthographically onto a plane which goes through the depth of the object's centroid followed by a perspective projection onto the image plane under uniform scaling, ${ }^{24}$ see Fig. 6 . However, the parallel projection falls into the generalized category of the affine camera. It corresponds to a projective camera with its projection center at the infinity. In this work, a magnification-dependent projection model which smoothly switches from a perspective projection to an orthographic projection is developed. Let start with a finite projective camera model which can be written as: ${ }^{7}$

$$
\mathrm{P}_{0}=\mathrm{KR}[\mathrm{l}-\mathrm{C}]=K\left(\begin{array}{ll}
\overrightarrow{r_{1}} & -{\overrightarrow{r_{1}}}^{T} \mathbf{C} \\
\overrightarrow{r_{2}} & -{\overrightarrow{r_{2}}}^{T} \mathbf{C} \\
\overrightarrow{r_{3}^{T}} & -{\overrightarrow{r_{3}}}^{T} \mathbf{C}
\end{array}\right),
$$

where $\mathbf{C}$ is the position of the projection center, $R \in \bullet \bullet(3)$ is the orientation of the projection frame, $\vec{r}_{i}$ is the $i$ 'th row of $\mathrm{R}$ and $\mathrm{K}$ is the matrix of intrinsics of the form:

$$
\mathrm{K}=\left(\begin{array}{ccc}
a f & s & p_{x} \\
0 & a^{-1} f & p_{y} \\
0 & 0 & 1
\end{array}\right)
$$

where $f$ represents the focal length, $a$ denotes the ratio of the pixel dimension in $x$ and $y$ directions, $s$ is referred as the skew parameter which is null for most normal imaging systems, and $\left(p_{x}, p_{y}\right)^{T}$ are the coordinates of the principal point $e_{0}$. The principal ray of the imaging system is in the direction of the vector $\overrightarrow{r_{3}}$, and the value $d_{0}=-\vec{r}_{3}^{T} \mathbf{C}$ is the distance of the world origin from the camera center in the direction of the principal ray.

If the magnification of our imaging system is increased by a scale factor $g$, then the image plane is pushed toward the viewed object. This is equivalent to move the camera center backward along the principal ray for the scale factor $g$, so that the center of the camera is moved to $\mathbf{C}-g \overrightarrow{r_{3}}$. Replacing $\mathbf{C}$ by $\mathbf{C}-g \overrightarrow{r_{3}}$ in Eq. (8) gives the projection matrix at magnification $g$ :

$$
P_{g}=\mathrm{K}_{g}\left(\begin{array}{cc}
\overrightarrow{r_{1}} T & -\overrightarrow{r_{1}}\left(\mathbf{C}-g \overrightarrow{r_{3}}\right) \\
\overrightarrow{r_{2}} T & -\overrightarrow{r_{2}} T\left(\mathbf{C}-g \overrightarrow{r_{3}}\right) \\
\overrightarrow{r_{3}} T & -\overrightarrow{r_{3}} T\left(\mathbf{C}-g \overrightarrow{r_{3}}\right)
\end{array}\right)=\mathrm{K}_{g}\left(\begin{array}{cc}
\vec{r}^{T} & -{\overrightarrow{r_{1}}}^{T} \mathbf{C} \\
\overrightarrow{r_{2}} T & -{\overrightarrow{r_{2}}}^{T} \mathbf{C} \\
\overrightarrow{r_{3}} T & d_{g}
\end{array}\right),
$$

where the terms $\vec{r}_{i}^{T} \overrightarrow{r_{3}}$ are zeros for $i=1,2$ because $\mathrm{R}$ is a rotation matrix. The scalar $d_{g}=-{\overrightarrow{r_{3}}}^{T} \mathbf{C}+g$ is the depth of the world origin with respect to the imaging system center in the direction of the principal ray $\overrightarrow{r_{3}}$ of the imaging system.

The effect of zooming by a factor $g$ is to move an image point $\mathrm{x}^{u}$ on a line radiating from the principal point $\mathrm{e}_{0}$ to the point $\mathbf{x}^{\prime u}=g \mathbf{x}^{u}+(1-g) e_{0}$. from similar triangles we obtain that:

$$
g=\frac{f_{g}}{f_{0}}=\frac{d_{g}}{d_{0}} .
$$

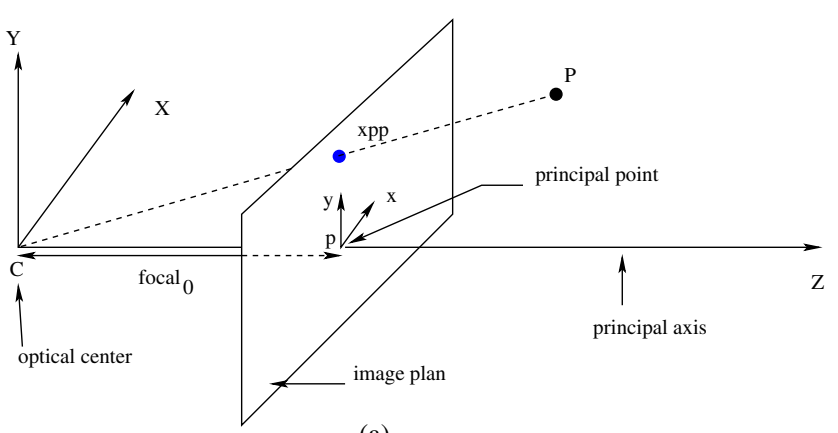

(a)

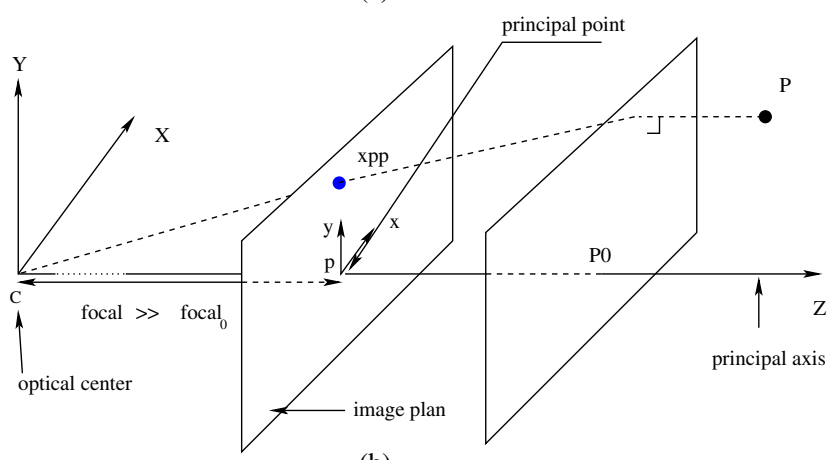

(b)

Fig. 6 (a) Perspective projection. (b) Weak perspective projection. When the focal length is much bigger when compared the distance 3-D scene and the image plane, the projection gets closer to an ideal orthographic projection. 
The resulting projection matrix at a magnification $g$ is:

$$
\begin{aligned}
P_{g} & =\mathrm{K}\left(\begin{array}{ccc}
g & 0 & 0 \\
0 & g & 0 \\
0 & 0 & 1
\end{array}\right)\left(\begin{array}{cc}
{\overrightarrow{r_{1}}}^{T} & -{\overrightarrow{r_{1}}}^{T} \mathbf{C} \\
\overrightarrow{r_{2}} & -{\overrightarrow{r_{2}}}^{T} \mathbf{C} \\
{\overrightarrow{r_{3}}}^{T} & d_{g}
\end{array}\right) \\
& =g \mathrm{~K}\left(\begin{array}{cc}
{\overrightarrow{r_{1}}}^{T} & -{\overrightarrow{r_{1}}}^{T} \mathbf{C} \\
\overrightarrow{r_{2}} & -{\overrightarrow{r_{2}}}^{T} \mathbf{C} \\
{\overrightarrow{r_{3}}}^{T} g^{-1} & d_{0}
\end{array}\right) .
\end{aligned}
$$

When $g \gg 1$, the third row of the projection matrix $g^{-1}{\overrightarrow{r_{3}}}^{T}$ tends to be small since $\left\|\overrightarrow{r_{3}}\right\|=1$. In this case the projection matrix corresponds to an instance of an affine camera. When $g \vec{\infty}$, the projection matrix corresponds to a parallel projection.

In the next paragraph, we describe how our development of static distortion model and projection model is embedded in a smooth magnification calibration model. A set of PDEs are established and solved for each calibration parameters.

\subsection{Static Calibration Method}

Let assume a calibration through the range $\left[g_{0}, g_{t}\right]$ of magnification. This interval is uniformly discretized within a $G$ sampling step. We assume also in here that the dynamic distortion calibration was done beforehand and that the image drift is corrected. The different steps of the static calibration can be summarized by the Algorithm 2 .

\section{Real Experimental Results}

The calibration method developed so far is validated on a JSM 820 SEM manufactured by JEOL, see Fig. 7. The electron gun is equipped with a tungsten filament that

Algorithm 2 PDEs estimation and solving.

1. for $g=g_{0}$ to $g_{f}$ with step $G$ do

Data: A set of $N$ image with grid points

2. calibrate the projection matrix assuming $\xi_{r}\left(g_{i}\right)=0$ and $\xi_{t}\left(g_{i}\right)=0$;

3. comptue $f\left(g_{i}\right), s\left(g_{i}\right)$ and $a\left(g_{i}\right), p_{x}\left(g_{i}\right)$, and $p_{y}\left(g_{i}\right)$. compute distortion center $e\left(g_{i}\right)$ as explained in Sec. 3.1.2;

4. if $e\left(g_{i}\right)$ is not degenerate then

5. initialize $\xi_{t}$ and $\xi_{r}$ to zero and estimate $\xi_{t}\left(g_{i}\right)$ and $\xi_{r}\left(g_{i}\right)$ using bundle adjustment method $^{22}$ to refine all the parameters;

6. else

7. set $e\left(g_{i}\right)$ to the principal point and $\xi_{r}\left(g_{i}\right)=0$;

8. initialize $\xi_{t}$ to zero and the estimate $\xi_{t}\left(g_{i}\right)$ using bundle adjustment method $^{22}$ to refine all the parameters;

9. end

10. compute $\xi_{t}, \xi_{r}, e, f, s, a, p_{x}, p_{y}$ with respect to $g$ using PDA (see Appendix A for solving method).

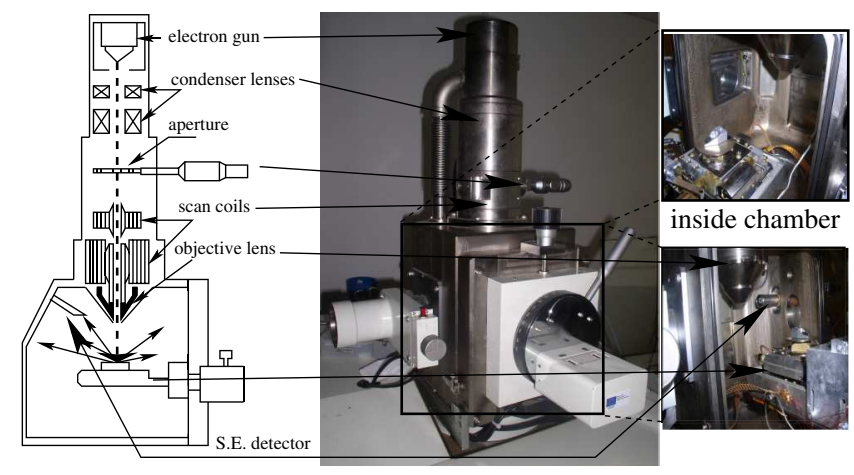

Fig. 7 The JSM 820 SEM manufactured by JEOL.

can support from 0.3 up to $30 \mathrm{kV}$ of acceleration voltage. During all the experiments the SEM images have a size of $512 \times 512$ pixels. The acceleration voltage is $15 \mathrm{Kev}$, the scan rate is 15 frames per second and the number of scans averaged is 8 . To evaluate the proposed calibration model, Algorithms 1 and 2 are run. The drift calibration is computed from $g_{0}=100 \times$ up to $g_{f}=30 \mathrm{~K} \times$. The static calibration model is computed from $g_{0}=100 \times$ to $g_{f}=10 \mathrm{k} \times$ with a step of $500 \times$. The obtained results are described and commented in the next sections.

\subsection{Static Calibration of the JEOL-JSM 820}

At this step, the SEM's images are supposed to be shifted back and corrected regarding to the dynamical pixel-drift. The static calibration is carried out using a multi-scale planar grid designed in our laboratory for our method of calibration, see Fig. 2. The planar grid contains regular chess-board squared regions with different sizes: $25 \mu \mathrm{m}, 10 \mu \mathrm{m}, 5 \mu \mathrm{m}$, $2 \mu \mathrm{m}$, and $1 \mu \mathrm{m}$, see Fig. 8. The grid calibration points $\left\{\mathbf{x}_{i}^{c}\right\}_{i \in \bullet}$ are manually selected and 5 images are taken for each magnification scale $g_{i} \in[100 \times, 10 k \times]$ within a step $G=500 \times$ (see Fig. 9). This step was chosen experimentally so that the least square error of fitting PDA is as small as possible. A minimum of 8 points per image are needed to ensure stable estimation. The static calibration follows the procedure explained in algorithm.

\subsubsection{Evidence of presence of distortion}

The estimation of the center of distortion shows an exponential behavior. It is almost linear at low magnification and strongly nonstable at high magnification. The break point appears as being located at $5 \mathrm{k} \times$. Across the scales of stability the distortion parameters appear as having an exponential dynamic. The static distortion effect vanishes when one comes close to the magnification break point, Fig. 10. Applying Algorithm 2, gives rise to the following PDEs for the trajectory of the static distortion center:

$\begin{array}{cccc} & \mathbf{P D E} & \mathbf{g}_{\mathbf{1}}=\mathbf{1 0 0} \times & \mathbf{g}_{\mathbf{2}}=\mathbf{1 0 k} \times \\ e_{x} & \ddot{e}_{x}=-0.02 e_{x} & 230.21 & 225.08 \\ e_{y} & \ddot{e}_{y}=-0.02 e_{x} & 270.34 & 265.10\end{array}$

and solutions are: 


$$
\begin{gathered}
e_{x}(g)=230+10\left[1-\exp \left(2 \cdot \frac{g}{10^{6}}\right)\right] \\
e_{y}(g)=270+10\left[1-\exp \left(2 \cdot \frac{g}{10^{6}}\right)\right] .
\end{gathered}
$$

Figure 10 shows the solutions.

\subsubsection{Distortion model}

The estimation of $\xi_{r}(g)$ and $\xi_{t}(g)$ with PDA show an exponential behavior of both parameters. With increasing magnifications, $\xi_{r}(g)$ tends to vanish and $\xi_{t}(g)$ becomes important. This observation is related to the fact that at high magnification (more than $5 \mathrm{k} \times$ ), the dynamic drift becomes important for each pixel and is not global between frames. Thus, a global time shift is compensated by the dynamical drift estimation and a local pixel-shift is estimated by the tangential component of the distortion model. Applying Algorithm 2, gives rise to the following PDEs concerning the static distortion parameters:

$$
\begin{array}{llcc} 
& \mathbf{P D E} & \mathbf{g}_{\mathbf{1}}=\mathbf{1 0 0 \times} & \mathbf{g}_{\mathbf{2}}=\mathbf{1 0 k} \times \\
\xi_{r} & \ddot{\xi}_{r}=-0.0086 \dot{\xi}_{r}+0.015 \xi_{r} & 0.003 & 0.002 \\
\xi_{t} & \ddot{\xi}_{t}=-0.0042 \dot{\xi}_{t}+0.0007 \xi_{r} & -7 \cdot 10^{-7} & -59 \cdot 10^{-8}
\end{array}
$$

and solutions are:

$$
\begin{aligned}
& \xi_{r}(g)=0.003-13 \cdot 10^{-12} g-6 \cdot 10^{-9} g^{2} \\
& \xi_{t}(g)=-7 \cdot 10^{-7}-13 \cdot 10^{-13} g+6 \cdot 10^{-13} g^{2}
\end{aligned}
$$

Figure 11 shows the solutions.

\subsubsection{Projection model}

Applying Algorithm 2 gives rise to the following PDEs for the intrinsic parameters of the projection model:

$$
\begin{array}{ccc}
\text { PDE } & \mathbf{g}_{\mathbf{1}}=\mathbf{1 0 0} \times & \mathbf{g}_{\mathbf{2}}=\mathbf{1 0 k} \times \\
\dot{f}-0.35 \cdot 10^{-4}=0 & 0.03 \cdot 10^{4} & 0.35 \cdot 10^{4} \\
10^{13} \ddot{a}=-0.2225 \dot{a}+0.0017 a & 1 & 1.1 \\
10^{13} \ddot{s}=-0.2059 \dot{s}+0.0016 s & 10^{-3} & 1.9 \cdot 10^{-3}
\end{array}
$$

and solutions are:

$$
\begin{aligned}
& f(g)=0.35 \cdot 10^{-4} g \\
& a(g)=0.05\left(g \cdot 10^{-4}\right)+0.95\left(g \cdot 10^{-4}\right)^{2} \\
& s(g)=\frac{-0.05+0.65\left(g \cdot 10^{-4}\right)+0.40\left(g \cdot 10^{-4}\right)^{2}}{10^{3}} .
\end{aligned}
$$

Figure 12 depicts the solutions. The principal point can be assumeed as being independent of the magnification. Indeed, the obtained calibration results exhibit bare variation of some 10 pixels around a median principal point of $(245,260)^{T}$ pixel. The camera center trajectory has a uniform variation with respect to the magnification variation. Finally, Fig. 13 shows the RMS reprojection error between image points and back-projected grid points. Whereas it corresponds to a median value of 7 pixels without any correction and to 3 pixels after drift compensation and without distortion correction, it decreases down to 1.45 pixel with both drift and distortion correction.

\subsection{Discussion and Comparison to State-of-the-Art Methods}

The obtained calibration results can be discussed as follows:

(1) The fitting error of the distortion center has an std (standard deviation) of 7 pixels (see Fig. 10). Also the fitting error in distortion parameters have stds of $2 \cdot 10^{-4}$ and $3 \cdot 10^{-7}$ for the first and second distortion parameter, respectively (see Fig. 11). These amounts of errors are acceptable as was confirmed by the reprojection error of 0.9 pixels. Also these calibration results allows us to reach a good level of accuracy in the measurement of deformation of a cantilever and in 3-D reconstrucition of a nano-ball as will be shown in next paragraphs. Apart from these experiments, we also tried 3rd and 4th order of PDA and the results were worse. This instability at higher order is mainly due to the noise that is introduced by the computation of 3rd and 4th order derivatives using discrete data. An over fitting with 3rd or 5 th order Legender is also not a good practical solution because it over fits the calibration data, it introduces a lot of oscillation, and then it will fail on test data. In similar context, the second order model has been also used with success in Ref. 6 to model the pixel distortion phenomenon of an FEM (force electron microscope). The 2 nd order PDA is a good tradeoff between fitting and stability with test data. In the
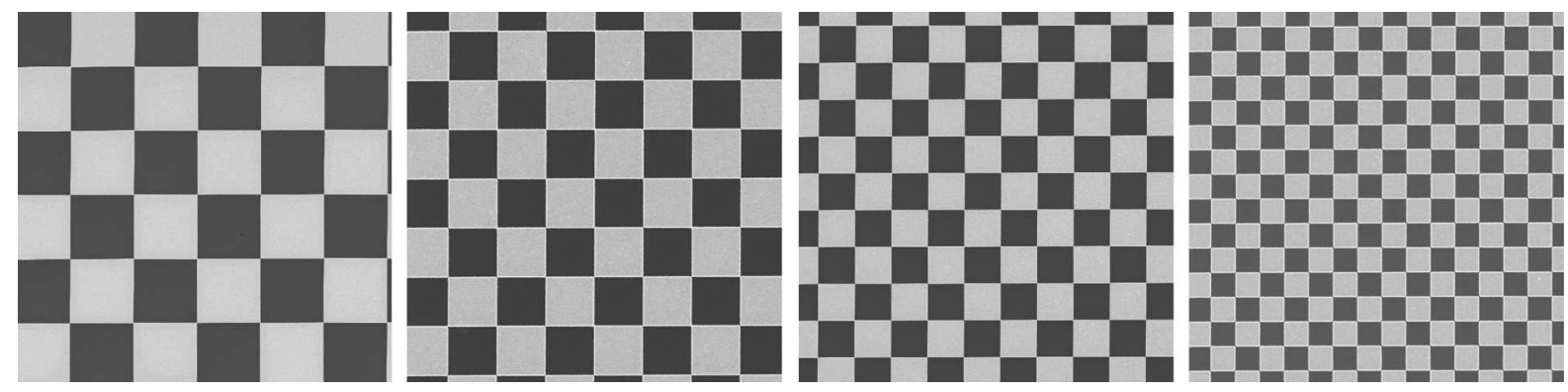

Fig. 8 From left to right: Square side sizes are, respectively of $25 \mu \mathrm{m}, 10 \mu \mathrm{m}, 5 \mu \mathrm{m}$, and $1 \mu \mathrm{m}$. 


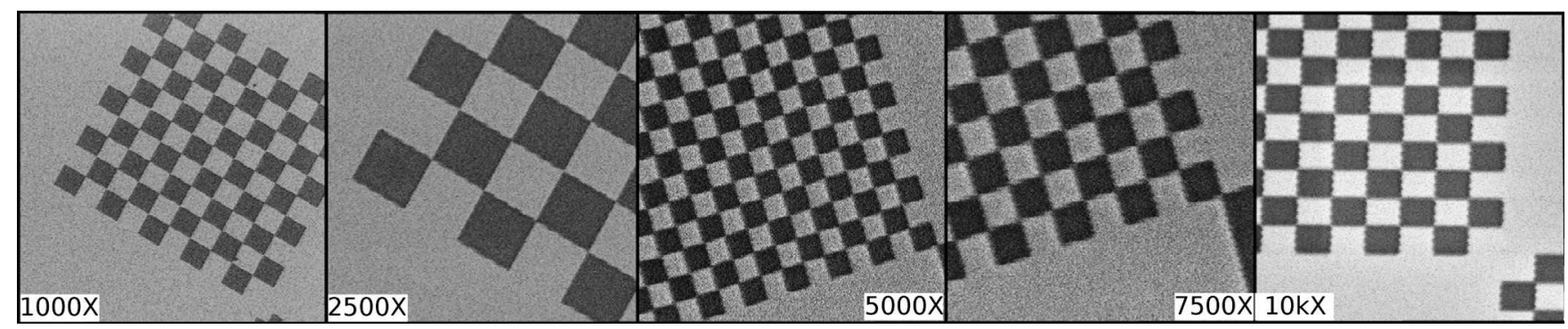

Fig. 9 Images of chess grids at different magnifications. The square corners are used for calibration. A minimum of 8 points per image are needed to ensure stable estimation.
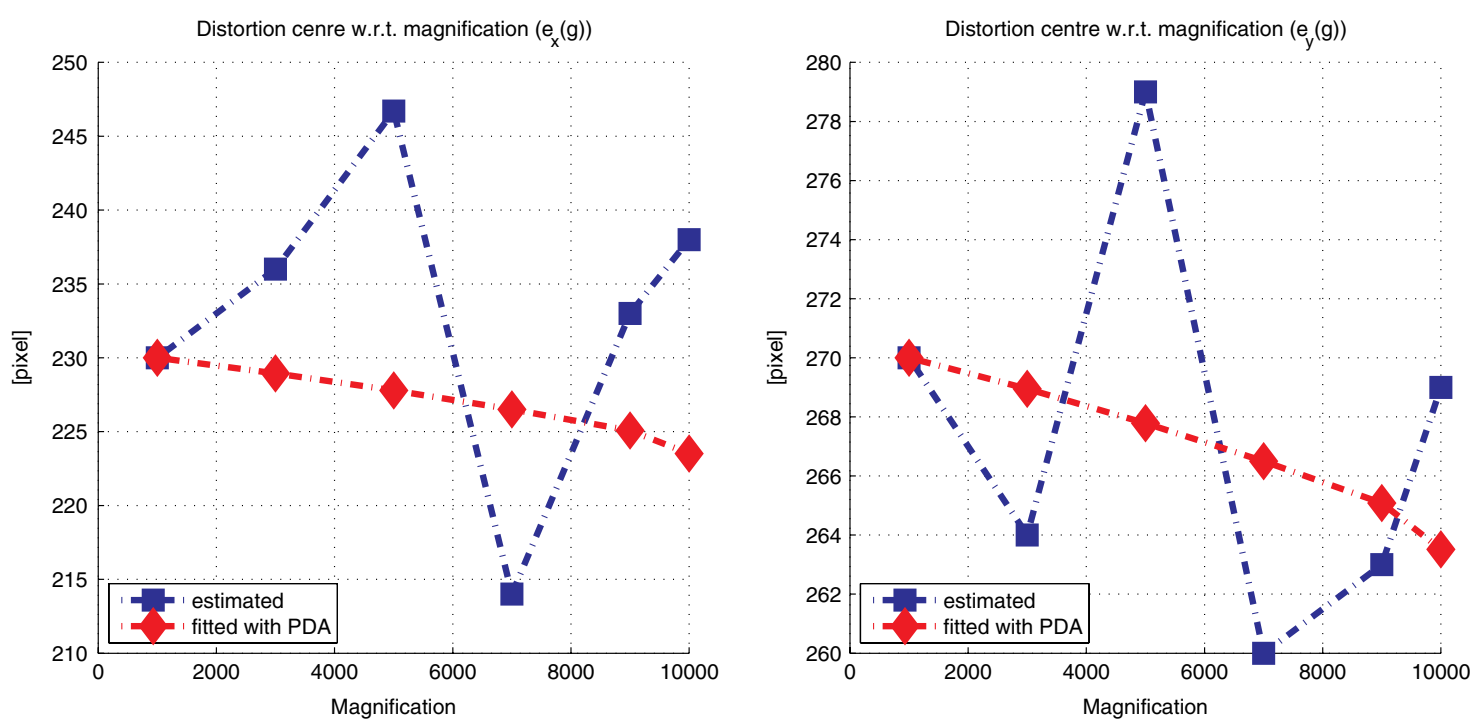

Fig. 10 Variation of the distortion center in $x$ - and $y$-axis with respect to magnification factors.

case of a long run time experiment where the pixeldrift becomes important, our 2nd order PDA model can be easily supplemented with an extended Kalman filter as was used in Ref. 6 to compensate the accumulated errors.

(2) In the set of discrete calibrated magnification scale factor, our method has similar performance to the reference state-of-the-art method. ${ }^{2}$

(3) In intermediate magnification without direct calibration, our method proposes a solution through the embedded smooth magnification-time-space model and provides calibration parameters.

\subsection{Application to Cantilever Deformation Measurement}

To show the relevance of our approach, we compare the measures of the same deformation applied to a cantilever at three different magnification factors $6450 \times, 7760 \times$, and $8890 \times$. This experiment will provide an estimation of the repeatability and the accuracy of the measurements compared to a reference measure. The reference measure uses calibration parameters computed directly at the magnifications $6450 \times, 7760 \times$, and $8890 \times$ using the method proposed in Ref. 2. The deformation measures which evaluate our calibration method use the magnification-continuous calibration parameters described in the previous section. It is worthy to notice that these parameters were not computed directly at these three magnification scales but the computed magnification-continuous functions allows us to find the calibration parameters at any magnification factor in the range $[100 \times, 10 \mathrm{k} \times]$.

The cantilever is $35 \mu \mathrm{m}$ length, $3.5 \mu \mathrm{m}$ width, and $300 \mathrm{~nm}$ thick. The experimental setup consists on a Kleindiek MM3A-EM micro-manipulator with a planar surface mounted on the tip. A cantilever is fixed within a holder and is deformed with the contact of the planar surface which is actuated by the arm of the MM3A-EM, see Fig. 14. Such an experiment may have several applications in the mechanical characterization of cantilevers, ${ }^{25}$ of biological deformable objects, ${ }^{26}$ the analysis of structured surfaces, ${ }^{27}$ etc.

A set of 11 configurations are taken for each magnification factor $(11 \times 3$ images $)$. Initially the cantilever is straight and free from any contact with the planar surface. After that, it comes close to the tip of the cantilever which is progressively pushed forward by the MM3A-EM. After $7 \times 3$ acquired images at different configurations of the deformation, the cantilever is progressively dragged backward to the initial contact-free configuration, see Fig. 15. Through the whole experiment, a time tracking frame acquisition is 

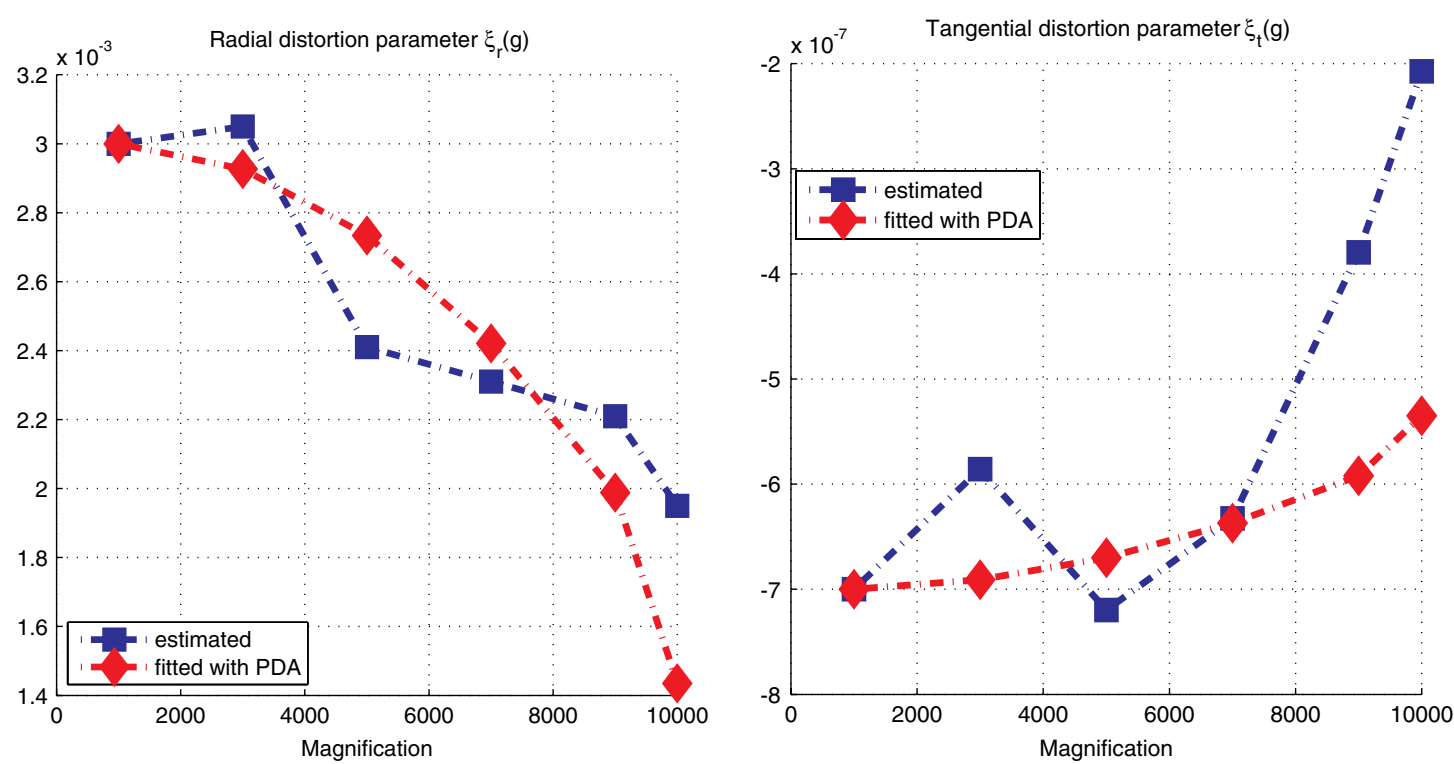

Fig. 11 Variation of the distortion parameters with respect to magnification scale. Left: The radial distortion parameter $\xi_{r}$. Right: The tangential distortion parameter $\xi_{t}$.
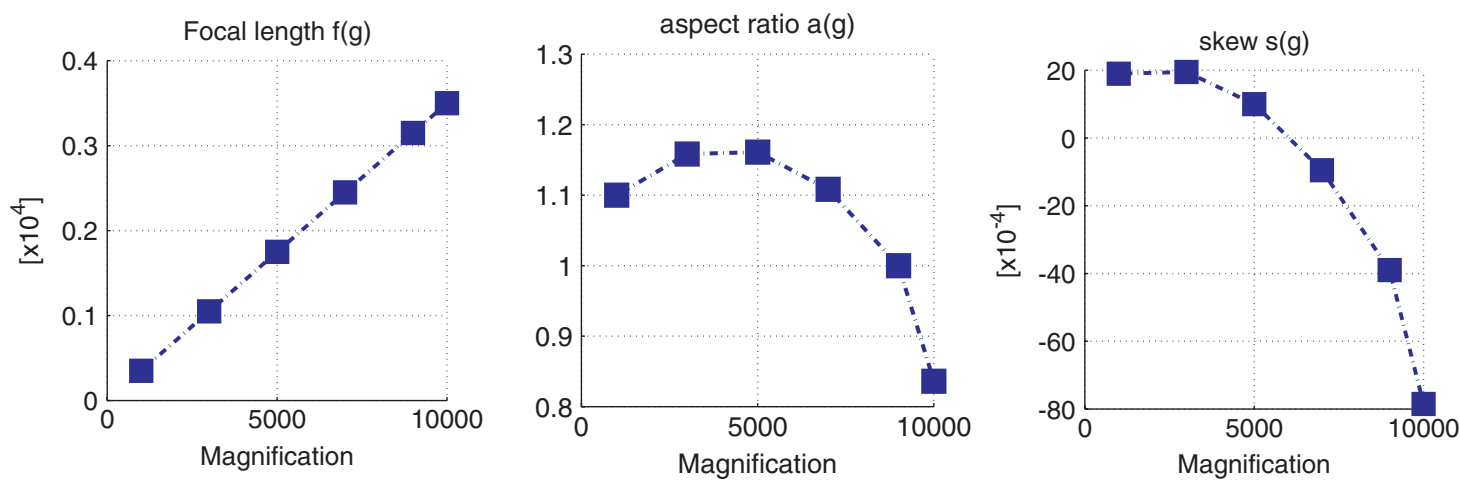

Fig. 12 Projection matrix parameters with respect to magnification scale. Left to right: focal length, aspect ratio, and skew.

automatically processed using the PC processor's clock trigger. This time acquisition is important to retrieve the amount of pixel by which the acquired images have drifted.

For each magnification factor the deformation measurements are assessed as follows:

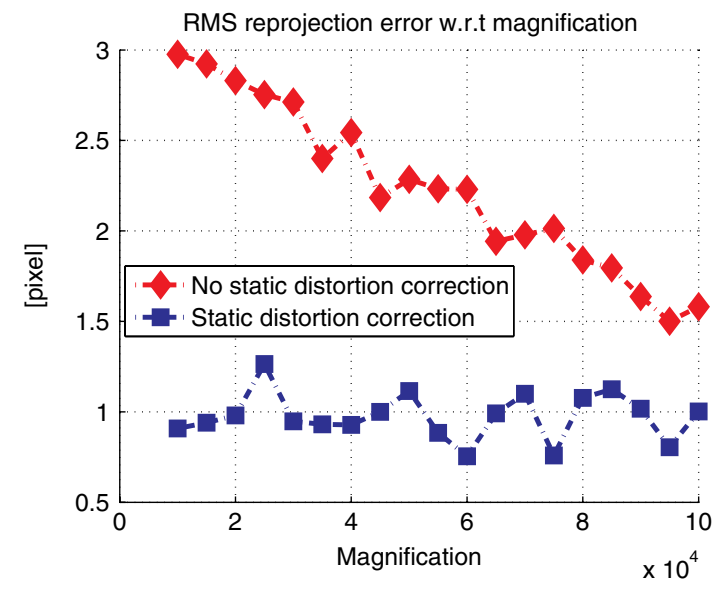

Fig. 13 The RMS reprojection error through magnification scales. The average RMS error is about 0.9 pixels on average.
Step 1: According to the estimated parameters of the pixel-drift behavior, the 11 acquired images are drift-compensated using Ref. 2.

Step 2: According to the estimated parameters of the static pixel distortion, the 11 acquired images are undistorted using our proposed static distortion model and Ref. 2 for the reference measures.

Step 3: The cantilever deformation is fitted with a 2 nd order polynomial curve.

Step 4: The deformed tip of the cantilever is tracked through the images by taking into account the constant length of the cantilever and its base tip since it is an isometric deformation.

Step 5: During the deformation, the cantilever sweeps a virtual plane. The affine homography between image pixels and this deformation plane is assessed ${ }^{8}$ by taking into account the estimated parameters of our proposed projection model and the projection model in Ref. 2 for the reference measures, see Fig. 16 right. The Euclidean stratification is done using the length of the cantilever provided by the manufacturer.

The measured deformation reach a maximum of $250 \mathrm{~nm}$. After drift and distortion correction, the standard deviation of 


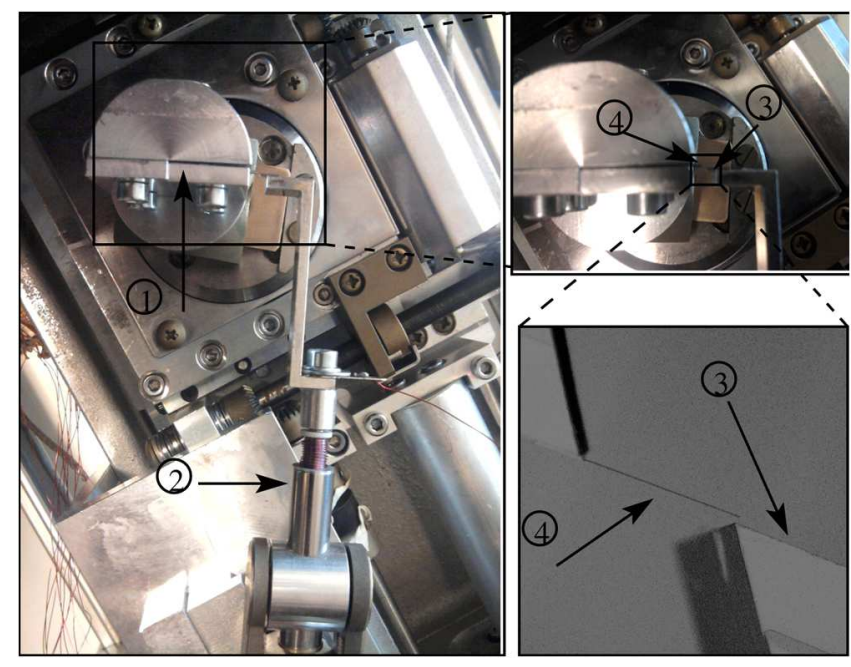

Fig. 14 The experimental setup: 1. the holder of the cantilever; 2 . the Kleindiek MM3A-EM micro-manipulator; 3. the planar surface; and 4. the cantilever to be deformed.

the error among the three scales is of about $10 \mathrm{~nm}$ which is an acceptable amount of error at this scale of magnification (see Fig. 16).

\subsection{3-D Reconstruction of a Nano-Ball}

In SEM imaging system it is difficult to have the whole observed specimen in one single image. We need different images at different scales to have local information with details as well as global information. In order to register all this information to obtain a 3-D shape with coarse to fine representation we need a magnification-continuous calibration model. The final experiment is a 3-D reconstruction of a ball of $900 \mathrm{~nm}$ diameter. This ball is attached to the tip of the cantilever which was used in the previous experiment. The ball is moved with the Kleindiek micro-manipulator to have multiple views. Also, the views go from $9 \mathrm{k} \times$ to $10 \mathrm{k} \times$ within a step of $100 \times$. At the end a sequence of $2 \cdot 10^{4}$ frames are gathered at $10 \mathrm{fps}$. The dynamical drift is corrected with the method proposed in Ref. 2 . The calibration model we developed is then used to undistort the frames and a KLT tracker ${ }^{28}$ together with SIFT feature detector ${ }^{29}$ are used to follow feature points through the acquired frames [see Fig. 17(a)]. Using the proposed projection model, we estimate the fundamental matrix between consecutive views from the point tracks. From the set of fundamental matrices, we then compute an affine reconstruction, upgrade it to metric and finally launch bundle adjustment to finely tune the reconstruction This process outputs 2643 -D points $\left(x_{j}, y_{j}, z_{j}\right), j=1, \ldots, 264$ [see Fig. 17(b)]. We then reconstruct a dense 3-D surface from the point cloud. Assuming that the surface is smooth and well represented by the point cloud, this is achieved by a moving least squares reconstruction. ${ }^{30}$ The surface is triangulated to form a mesh with 502 faces and 264 vertices [see Fig. 17(c)]. The 3-D reconstructed geometric model of the nano-ball can be used for instance in geometric characterization, in automated nanomanipulation or visual servoing applications.

\section{Conclusion}

In this paper we have presented a magnification-continuous model for static and projection calibration of the imaging

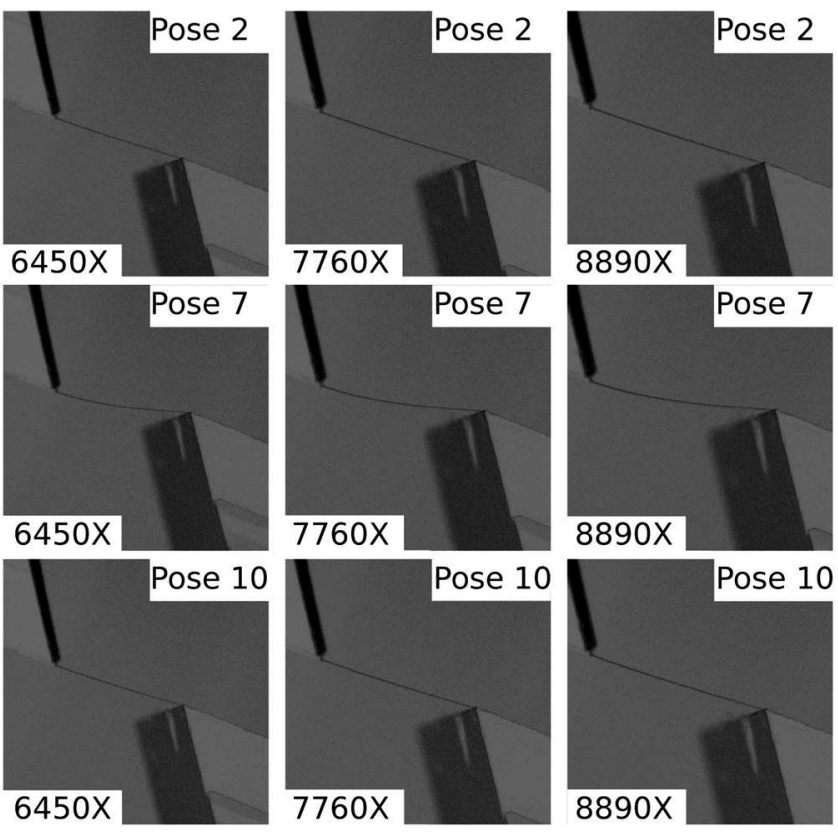

Fig. 15 Three configurations of deformation (pose 2, 7, and 10) at the three different magnification factors. The maximum of the deformation is at pose 7 . Poses 1 and 11 are contact-free between the cantilever and the plane.

system of the SEM. The developed model is applied to calibrate the JEOL-JSM 820 SEM. We proposed two real experiments to show the usefulness of our calibration model. Recent works with pressure controlled SEM suggest an existing influence of the pressure on the drift and the spatial distortion especially at very high magnifications. ${ }^{31}$ In future work, we intend to introduce the pressure as a third variable and quantify its impact. Henceforth, it will be challenging to validate our model with further experiments at very high magnification scales.

\section{Appendix A: PDEs Estimation and Solving}

PDA is a term used by Ramsay ${ }^{32}$ to describe a parameter estimation method wherein coefficients of linear, possibly time varying, ordinary differential Equations (ODEs) are fitted empirically from data. Ramsay called his technique principal differential analysis because of analogies to principal component analysis (PCA), in which empirical linear algebraic-equation models are fitted using multivariate data. Ramsay and Silverman ${ }^{33}$ focused their efforts on problems in which dynamic systems respond to unknown, empirical, time-varying forcing functions. PDA has been used to fit linear differential equation models for a diverse array of applications including handwriting analysis, ${ }^{34}$ analysis of the movement of the lips during speech, ${ }^{35}$ economic modeling, ${ }^{36}$ and meteorological modeling. ${ }^{8}$

When describing PDA for linear PDE models, Ramsay and Silverman ${ }^{33}$ view the system dynamics as a linear differential operator (LDO) acting upon the process variables. For example, let $\mathbf{x}\left(g_{i}\right)$ be the discrete estimated calibration parameter which varies with respect to the magnification $g_{i}$ from $g_{0}$ until $g_{f}$. Let $D^{m} \mathbf{x}$ be the $m$ th derivative of the function $\mathbf{x}(g)$ with respect to $g$. The function $\mathbf{x}(g)$ is assumed to 


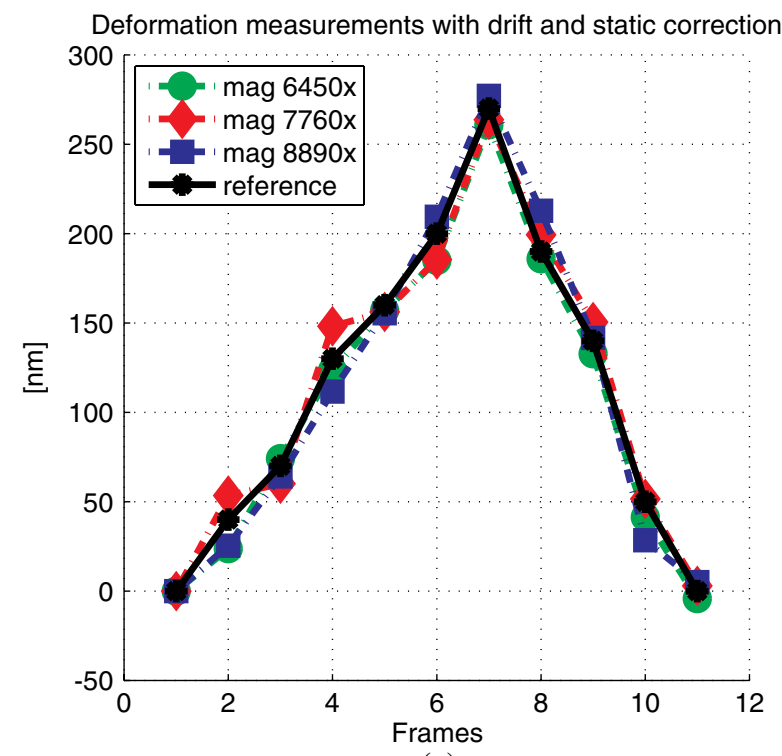

(a)

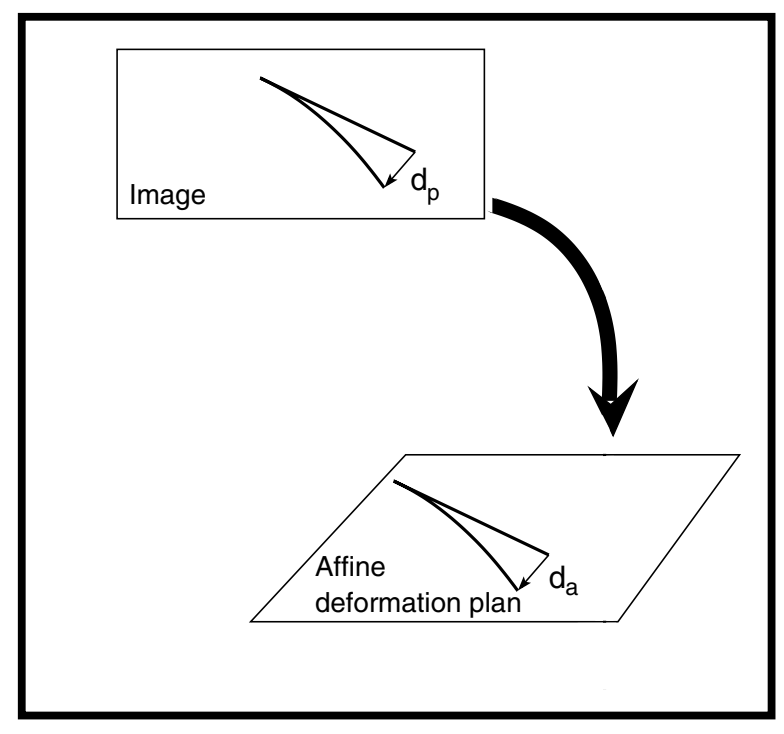

(b)

Fig. 16 (a) Comparison of the repeatability of our calibration model with the reference model. The calibration parameters using our model are evaluated using the magnification-continuous model calibrated in the previous experiment. The parameters of the reference model are estimated directly in the magnifications $6450 \times, 7760 \times$, and $8890 \times$. Deformation measures for the three magnification scales with drift and static distortion correction. The repeatability is of $10 \mathrm{~nm}$ and it can be seen that our model reaches a good accuracy and repeatability. (b) The measured deformation from the initial configuration to the bended configuration. The homography $H$ transforms the pixel deformation $d_{p}$ to an affine deformation $d_{a}$. The scale factor from affine to Euclidean measurement is assessed thanks to the length of the cantilever provided by the manufacturer ( $35 \mu \mathrm{m})$.

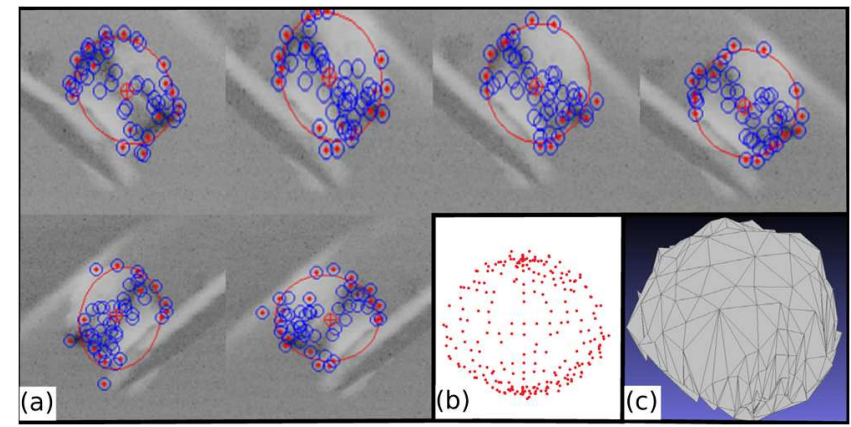

Fig. 17 3-D reconstruction of nano-ball of $900 \mathrm{~nm}$ diameter. (a) Some acquired frames and tracked points. (b) The 3-D point cloud of the nano-ball. (c) The 3-D reconstructed model of the nano-ball meshed in 264 vertices and 502 triangular faces.

be square integrable which is a valid assumption regarding to the fact that the calibration parameters are not degenerate. In this research work, we assume the identification of a second order LDO which determine the first (speed) and second (acceleration) order parameters of the calibration parameters:

$$
L=w_{0}^{x}+w_{1}^{x} D+D^{2}
$$

that comes as close as possible to satisfy the homogeneous linear differential equation:

$$
L \cdot \mathbf{x}=0 .
$$

In other words, if we compute the first and second derivatives $D \mathbf{x}$ and $D^{2} \mathbf{x}$ of $\mathbf{x}(g)$ with respect to magnification using finite differences, the operator $L$ annihilates the function $\mathbf{x}(g)$ as nearly as possible. Thus, we seek a linear differential equation model so that our data satisfies:

$$
D^{2} \mathbf{x}=-w_{0}^{x} \mathbf{x}-w_{1}^{x} D \mathbf{x}
$$

to the best possible degree of approximation. To carry out PDA, we adopt a least squares approach to the fitting of the differential equation model. The fitting criterion is to minimize, over $\left(\begin{array}{ll}w_{0}^{x} & w_{1}^{x}\end{array}\right)$, the sum of squared norms:

$$
J=\left\|\mathrm{A}\left(\begin{array}{c}
w_{0}^{x} \\
w_{1}^{x}
\end{array}\right)+b\right\|^{2},
$$

where

$$
\mathbf{A}=\left[\begin{array}{cc}
\mathbf{x}\left(g_{0}\right) & D \mathbf{x}\left(g_{0}\right) \\
\vdots & \vdots \\
\mathbf{x}\left(g_{f}\right) & D \mathbf{x}\left(g_{f}\right)
\end{array}\right], \mathbf{b}=\left[\begin{array}{c}
D^{2} \mathbf{x}\left(g_{0}\right) \\
\vdots \\
D^{2} \mathbf{x}\left(g_{f}\right)
\end{array}\right]
$$

A is a matrix of $\frac{g_{f}-g_{0}}{G}$ rows and 2 columns and is always of rank 2 . b is a vector of $\frac{g_{f}-g_{0}}{G}$ elements. The solution of such an over-determined least square problem is given as:

$$
\left(\begin{array}{l}
w_{0}^{x} \\
w_{1}^{x}
\end{array}\right)=\left(\mathrm{A}^{\top} \mathrm{A}\right)^{-1} \mathrm{Ab} .
$$

Solving Eq. (21) for each calibration parameter: $\xi_{t}, \xi_{r}, e, f, s$, $a, p_{x}, p_{y}$ give rise to PDEs that can be easily solved ${ }^{32}$ to obtain smooth magnification-dependent parameters. The step parameter $G$ is chosen experimentally so that we obtain the lowest least square error. It is worthy to notice that the magnification-continuous framework is not only a smoothing of a set of discrete set of parameters. Indeed, the established PDEs depend strongly of the development of static distortion models and projection models described in the previous sections. 


\section{References}

1. M. A. Sutton et al., "Metrology in a scanning electron microscope: theoretical developments and experimental validation," Meas. Sci. Technol. 17(10), 2613-2622 (2006).

2. M. A. Sutton et al., "Scanning electron microscopy for quantitative small and large deformation measurements part I: SEM imaging at magnification from 200 to 10,000," Exp. Mech. 47(6), 775-787 (2007).

3. N. Li et al., "Full-field thermal deformation measurements in a scanning electron microscope by 2D digital image correlation," Exp. Mech. 48(5), 635-646 (2008)

4. T. Zhu et al., "Quantitative stereovision in a scanning electron microscope," Exp. Mech. 51(1), 97-109 (2011).

5. P. Cizmar, A. E. Vladar, and M. T. Postek, "Real-time image composition with correction of drift distortion," Tech. Rep. arXiv:0910.0213 (2009).

6. B. Mokaberi and A. A. G. Requicha, in ICRA, pp. 416-421, IEEE Computer Society, New Orleans, LA (2004).

7. R. I. Hartley and A. Zisserman, Multiple View Geometry in Computer Vision (2nd edition), 2nd ed., Cambridge University Press, United Kingdom (2004).

8. Y. Ma et al., An Invitation to 3-D Vision: From Imagesto Geometric Models, SpringerVerlag, Germany (2003).

9. F. Vignon et al., in Physics in Signal and Image Processing, pp. 23-24, Office national d'études et de recherches aérospatiales, Châtillon, France (2001).

10. O. Sinram et al., in ISPRS Commission V Symposium, pp. 210-215, Corfu, Greece (2002).

11. N. Cornille, Accurate 3-D Shape and Displacement Measurement using a Scanning Electron Microscope, Ph.D. Thesis, University of South Carolina (USA) and Institut National des Sciences Appliquees, France (2005).

12. M. Jähnisch and S. Fatikow, "3-D vision feedback for nanohandling monitoring in a scanning electron microscope," Int. J. Optomechat. 1(1), 4-26 (2007).

13. S. Fatikow et al., "Visual feedback methods for nanohandling automation," Int. J. Inform. Acquis. 6(3), 159-169 (2009).

14. M. A. Sutton et al., "Scanning electron microscopy for quantitative small and large deformation measurements Part II: experimental validation for magnifications from 200 to 10,000," Exp. Mech. 47(6), 789-804 (2007).

15. T. Sievers and S. Fatikow, "Real-time object tracking for the robotbased nanohandling in a scanning electron microscope," J. Micromechat. 3(3), 267-284 (2006)

16. B. E. Kratochvil, L. Dong, and B. J. Nelson, "Real-time rigid-body visual tracking in a scanning electron microscope," Int. J. Robot. Res.28(4), 498-511 (2009).

17. K. Takeshi et al., "Image-based autonomous micromanipulation system for arrangement of spheres in a scanning electron microscope," Rev. Sci. Instrum. 75(6), 2033-2042 (2004).

18. T. Sievers and S. Fatikow, in IEEE/RSJ International Conference on Intelligent Robots and Systems, RSJ/ IEEE Computer Society, Japan (2005).

19. J. Goldstein et al., Scanning Electron Microscopy and X-ray Microanalysis, Kluwer Academic/Plenum Publishers, New York (2003).

20. R. Hartley and S. Kang, "Parameter-free radial distortion correction with center of distortion estimation," IEEE Trans. Pattern. Anal. Mach. Intell. 29(8), 1309-1321 (2007).

21. J. Heikkila and O. Silven, in CVPR'97: Proceedings of the 1997 Conference on Computer Vision and Pattern Recognition (CVPR'97), p. 1106, IEEE Computer Society, Washington, DC (1997).

22. I. Lourakis and A. Argyros, "SBA: a software package for generic sparse bundle adjustment," ACM Trans. Math. Softw. 36(1), 1-30 (2009).

23. M. Ritter et al., in ISPRS Image Engineering and Vision Metrology, Remote Sensing and Photogrammetry Society, Dresden, Germany (2006).

24. G. Xu and Z. Zhang, Epipolar Geometry in Stereo, Motion and Object Recognition: A Unified Approach, Kluwer Academic Publishers, NY, ISBN 0-7923-4199-6 (1996).

25. G. Ionascu et al., in International Conference on Semiconductor, IEEE Computer Society, Japan (2009).

26. M. Boukallel et al., "Smart microrobots for mechanical cell characterization and cell convoying," IEEE Trans. Biomed. Eng. 54(8), 1536-1540 (2007).

27. J. Dejeu et al., "Adhesion forces controlled by chemical self-assembly and $\mathrm{pH}$ : application to robotic microhandling," Appl. Mater. Interfaces 1(9), 1966-1973 (2009).

28. J. Shi and C. Tomasi, "Good features to track," in IEEE Computer Society Conference on Computer Vision and Pattern Recognition, 1994. Proceedings CVPR '94, Seattle, WA, pp. 593-600 (1994).
29. D. G. Lowe, "Distinctive image features from scale-invariant keypoints," Int. J. Comput. Vis. 60(2), 91-110 (2004).

30. D. Levin, ,Geometric Model. Sci. Visual. 3, 37 (2003), http://www.math .tau.ac.il/ levin/mls03cor.pdf.

31. C. Arnoult et al., Microscopy: Science, Technology, Applications and Education. Microscopy series, Formatex (2011), http://www.formatex .info/microscopy4/1211-1218.pdf

32. J. O. Ramsay, "Principal differential analysis: data reduction by differential operators," J. Roy. Statist. Soc. B 58(3), 495-508 (1996).

33. J. O. Ramsay and B. W. Silverman, Functional data analysis, 2nd ed., Springer, New York (2005).

34. J. O. Ramsay, "Functional components of variation in handwriting," J. Am. Stat. Assoc. 95(449), 9-15 (2000).

35. J. Lucero, "Identifying a differential equation for lip motion," Med. Eng. Phys. 24(7), 521-528 (2002).

36. J. Lucero,'Functional data analysis of the dynamics of the monthly index of nondurable goods production," J. Econometr. 107(1-2), 327-344 (2002).

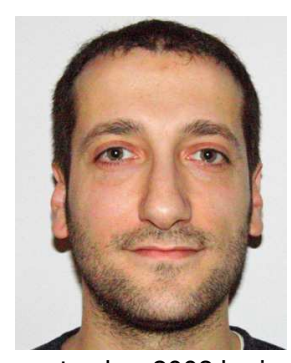

Abed C. Malti received the engineer degree from the University Abou-Bakr Belkaid (Tlemcen, Algeria) in 2001. He received the MS degree in control systems and signal processing from the National Polytechnic Institute of Lorraine (Nancy, France), in 2002. He received his $\mathrm{PhD}$ in the robotic team of LAAS-CNRS (Toulouse, France), in 2005 from the University of Paul Sabatier (Toulouse, France). He did a MS degree in basic mathematics during 3years up to 2008. Since september 2008 he is working as research fellow in computer vision. Currently, his main research interest is monocular non-rigid reconstruction.

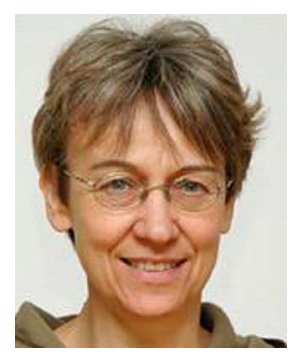

Nadine Le Fort-Piat is professor at ENSMM (National Institute in Mechanics and Microtechniques) and researcher at AS2M department of FEMTO-ST Institute. Her research areas concern Perception and Advanced Control strategies based on visual servoing and reinforcement learning for automation of microrobotic systems. The current projects concern the control of distributed microrobotic systems for robust and adaptive micromanipulation (ANR projects Smart Surface and SmartBlocks) and the imaging and visual servoing for automatic nanomanipulation under a SEM (ANR project Nanorobust).

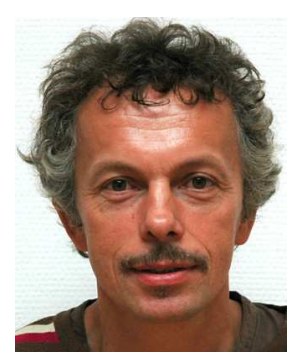

Patrick Rougeot was a senior technician in the development and control of robotic assembly equipment before receiving the graduate from the "Ecole Nationale Supérieure de Mécanique et des Microtechniques" (ENSMM), Besançon, France, in 1990. Then he is design engineer in 1996 in CNRS and is research engineer since 2000 in the department AS2M Femto-st. He works in the Micromanipulation, Micromechatronics, and Microrobotic Research Group in the field of Micro/Nanoforce measurement (SPECIMeN). His objectives are developments and exploitations of measurement chains at microand nanoscopic scales. His research interests are articulated around an atomic force microscope (AFM) for the characterization of the effects of surfaces and by design and developments of tools and sequences of micromanipulations. The major activities of his research concern the adhesion force measurement on structured and/or functionalized surfaces and polymer films.

Biographies and photographs of the other authors are not available. 